\title{
Hints of High Core Faraday Rotations from a Joint Analysis of VLBA and Optical Polarization Data
}

\author{
Juan Carlos Algaba* \\ University College Cork \\ E-mail: algaba@physics.ucc.ie \\ Denise C. Gabuzda \\ University College Cork \\ E-mail: gabuzda@physics.ucc.ie

\section{Paul S. Smith} \\ Steward Observatory, University of Arizona \\ E-mail: psmitheas.arizona.edu
}

\begin{abstract}
Although the continua of radio-loud Active Galactic Nuclei (AGN) are typically dominated by synchrotron radiation over virtually the entire spectrum, it is not clear whether the radio and higher-frequency emission originate in the same or different parts of the jet. Several different radio-optical correlations based on polarization data have been found recently, suggesting that the optical and radio polarization may be closely related, and that the corresponding emission regions may be cospatial[ $3,6,2]$. Our joint analysis of optical and VLBA polarization data for a sample of about 40 AGNs shows that, after correction for the inferred VLBA core Faraday rotations, most BL Lac objects and some quasars have aligned VLBA-core and optical polarizations, although many quasars also show no obvious relationship between their VLBA-core and optical polarization angles. This may indicate that not all AGNs have cospatial regions of optical and radio emission in their jets. However, another possibility is that some of the $7 \mathrm{~mm}-2 \mathrm{~cm}$ VLBA cores have Faraday rotations of the order of several tens of thousand of $\mathrm{rad} / \mathrm{m}^{2}$, which were not properly fit by our three-frequency data due to $n \pi$ ambiguities in the observed polarization angles, leading to incorrect subtraction of the effects of the core Faraday rotation, and so incorrect "zero-wavelength" radio polarization angles. The possibility of such high core Faraday rotations is supported by the results of the parsec-scale Faraday-rotation studies of Zavala \& Taylor[11] and Jorstad et al.[6].
\end{abstract}

The 9th European VLBI Network Symposium on The role of VLBI in the Golden Age for Radio Astronomy and EVN Users Meeting

September 23-26, 2008

Bologna, Italy

\footnotetext{
${ }^{*}$ Speaker.
} 


\section{Introduction}

The term Active Galactic Nucleus (AGN) refers to the existence of very energetic phenomena occurring in the centers of some galaxies that cannot be attributed to stars. In the standard model, AGNs consist of a black hole surrounded by an accretion disk. There is good evidence that relativistic jets in AGNs are powered by nonthermal synchrotron radiation; i.e., a flow of radiating, accelerated electrons embedded in a magnetic field, which dominates the continua of electromagnetic radiation over a wide window, extending from radio to soft $\mathrm{X}$-ray frequencies.

It is natural to suppose that all these wavebands should share common properties, and that variability should be correlated across the spectrum. However, consideration of the physics of AGN jets leaves it unclear whether this will, in fact, be the case. In some models [7], higher-energy emission is generated closer to the base of the jet, so that emission at different wavelengths arises in different regions of the jet with different physical properties (e.g. different magnetic-field configurations), while, in others, the high-energy and radio emission can be cospatial, at least for certain combinations of jet geometry and particle flow [4]. In addition, even if emission from various wavebands are intrinsically closely related, there are a variety of wavelength-dependent extrinsic processes, such as Faraday Rotation and scintillation, that can give rise to different observed properties at different wavelengths. Thus, it is important to correct for these extrinsic effects if we wish to test for correlated behaviour in different wavebands.

There is recent evidence of a much closer link between the radio and high-energy synchrotron radiation that was originally indicated by previous studies. For example, [5] found a tendency for gamma-ray flares to occur several months after the births of new VLBI component, suggesting these flares occur in these radio components, at appreciable distances down the jet. Polarization information has played a key role in revealing evidence for other correlations: [3] demonstrated a strong tendency for the simultaneously measured optical and Faraday-rotation-corrected VLBI core polarization angles to be aligned in about a dozen BL Lacs, and [6] observed similar behaviour for the optical and high-frequency radio polarization angles in a sample of highly polarized AGNs. Also, [2] observed a rapid, simultaneous rotation in the optical and 7mm VLBA-core polarization angles in 0420-014. All these results support the idea that the radio to optical, and even gamma-ray, emission may be more closely related than was previously thought.

\section{Observations and Results}

With the aim of verifying the results of [3] and searching for further evidence for opticalVLBI polarization correlations, we observed an additional $\sim 30$ AGNs, including BL Lac objects and both high- and low-polarization quasars (here, the degree of polarization refers to the optical waveband [8]), thus providing us with a sample of 40 AGNs for our analysis. We obtained $7 \mathrm{~mm}+1.3 \mathrm{~cm}+2 \mathrm{~cm}$ VLBA polarization data and nearly simultaneous optical polarization data with the Steward Observatory 2.3m telescope in three 24-hour sessions, on November 1, 2004, March 16, 2005 and September 26, 2005. The data reduction and imaging for the radio data were done with the NRAO Astronomical Image Processing System (AIPS) using standard techniques (see, e.g. [3]). 
The optical polarization observations spanned the VLBA observation runs (October 30-November 2, 2004; March 15-17, 2005; and September 25-29, 2005). These data were acquired using the SPOL imaging/spectropolarimeter [9]. On various nights, the instrument was configured for either imaging polarimetry using a KPNO "nearly mould" R filter (6000-7000A) or spectropolarimetry using a 600 line/mm diffraction grating. The data acquisition and reduction closely followed those described in[10]. The spectropolarimetric observations were averaged over the R-filter bandpass for direct comparison to the imaging polarimetry.

Since we have VLBA polarization data at three wavelengths, we expected to be able to correct for Faraday rotation in the region of the compact core, enabling a comparison between the "zero-wavelength" radio-core and optical polarization angles. If the optical and radio emission is cospatial, and the emitting region is optically thin at both wavebands, the difference $\Delta \chi$ between the optical $\chi_{o p t}$ and Faraday-rotation-free radio-core $\chi_{0}$ polarization angles should be close to zero. On the other hand, if the VLBI-core emission is optically thick at the observed radio wavelengths, this should give rise to an offset of $\Delta \chi=90^{\circ}$. Thus, our "null hypothesis" was that the distribution of $\Delta \chi$ values would be dominated by two peaks: one near $0^{\circ}$ and one near $90^{\circ}$. However, this was not the case for the new observations: although the complete sample of BL Lac objects displayed an overall clear peak near $0^{\circ}$, the quasars display a flatter distribution, possibly with a weak peak around $\sim 50^{\circ}[1]$. We discuss in section 3 the possible origins of this difference in behaviour shown by the BL Lac objects and the quasars in our sample.

\section{Discussion}

One obvious possibility is that there is indeed no correlation between the optical and VLBIcore polarization angles for both the high-polarization and low-polarization quasars. In other words, these results may provide evidence that the optical and VLBI-core emission in quasars is usually not cospatial, with the different emitting regions having different properties. If so, this would seem to indicate a difference between the geometries or physical conditions of the jets of quasars and BL Lac objects that is worthy of further study.

However, the hint of a possible weak peak in the $\Delta \chi$ distribution for the quasars suggests another possibility: that the VLBI cores are subject to internal Faraday rotation. In this case, the rotation of the radio polarization angle initially obeys the $\lambda^{2}$ law characteristic of external Faraday rotation, but then saturates at rotations of about $40-50^{\circ}$ with increase in the observing wavelength. If all three of our wavelengths were in this regime, this could appear as a small core Faraday rotation, leading to an inferred $\chi_{0}$ of about $40-50^{\circ}$ away from its true value. Our past investigation showed no evidence for enhanced depolarization in the quasar cores compared to the BL Lac cores, leading us to discard this idea as an explanation for the behaviour of the quasars in our sample as a whole [1].

There is, however, another possibility: that the $7 \mathrm{~mm}-2 \mathrm{~cm}$ quasar cores are subject to appreciably higher local but external Faraday rotations than the BL Lac cores at these wavelengths. This would be consistent with the tendency for quasar cores to have higher Faraday rotation measures than BL Lac cores at $2 \mathrm{~cm}-4 \mathrm{~cm}$ [11]. In fact, core rotation measures as high as tens of thousands of $\mathrm{rad} / \mathrm{m}^{2}$ have been reported previously $[6,11]$. In the case of high core rotation measures, we 


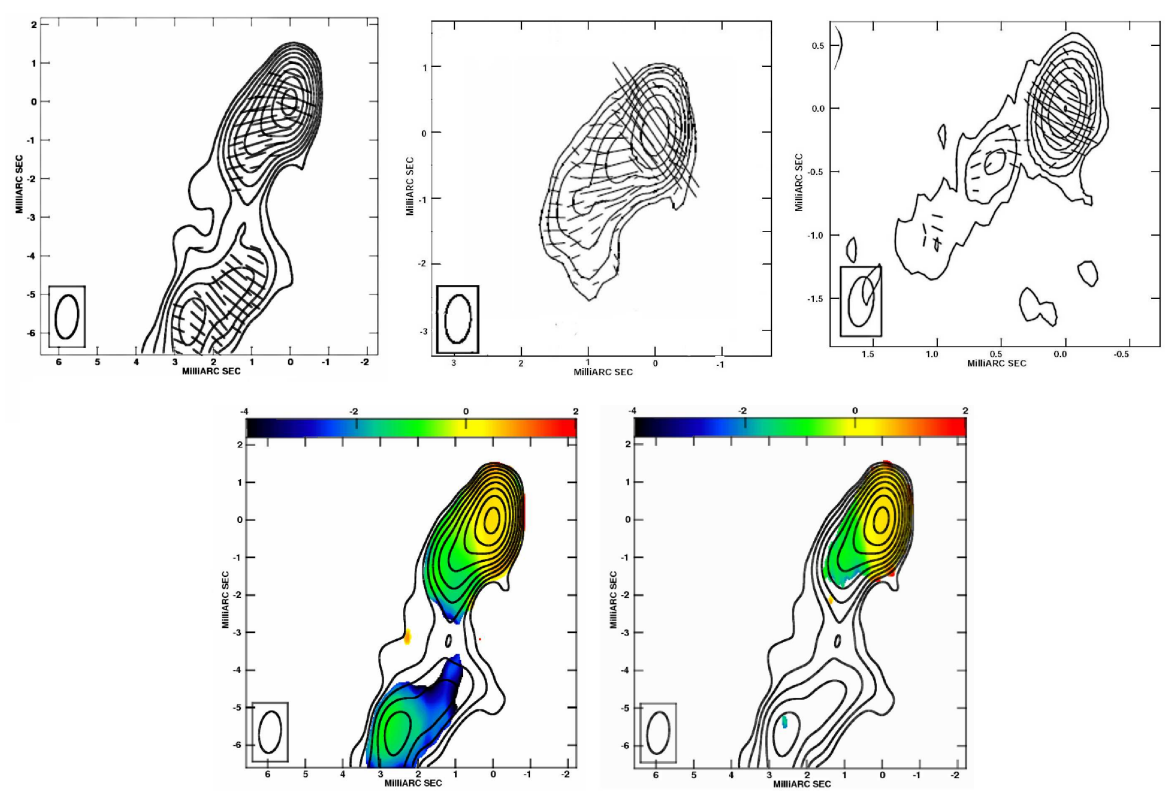

Figure 1: Top: total intensity maps with superposed polarization vectors for $2230+114$ at $2 \mathrm{~cm}$ (left), $1.3 \mathrm{~cm}$ (middle) and $7 \mathrm{~mm}$ (right). Bottom: $2 \mathrm{~cm}-1.3 \mathrm{~cm}$ (left) and $1.3 \mathrm{~cm}-7 \mathrm{~mm}$ (right) spectral-index distributions for this source; both core spectral indices are $\sim 0.46$, giving no evidence for a change in optical depth in the observed frequency range.

encounter problems with $n \pi$ ambiguities when fitting the rotation measures, leading to incorrect determinations of both the rotation measure and $\chi_{0}$.

Our data suggest that such ambiguities may be present. For example, if we observe a roughly $90^{\circ}$ change in the observed polarization angles between two radio wavelengths, it is natural to suppose that this is due to a transition between the optically thick and optically thin regimes. This can be verified by examining the observed spectral indices and degrees of polarization of the VLBI core. However, this supporting evidence is not found in most cases where we observe polarization-angle rotations by roughly $90^{\circ}$ between neighbouring wavelengths, suggesting that for these objects, high core Faraday rotations may be relevant. In addition, some of the core rotation measures indicated by our observations are low compared to the typical values deduced by [11], despite taking into account the fact that our $7 \mathrm{~mm}-2 \mathrm{~cm}$ observations probe somewhat smaller scales, where we expect both the electron density and magnetic-field strength to be higher.

Two examples are shown in Figs. 1 and 2. Fig. 1 shows our images for the quasar 2230+114, using the original $1.11 \times 0.58$ mas $15 \mathrm{GHz}$ beam to convolve all frequency maps. The nominal observed values for the polarization angles are $70^{\circ}$ at $2 \mathrm{~cm}, 34^{\circ}$ at $1.3 \mathrm{~cm}$ and $94^{\circ}$ at $7 \mathrm{~mm}$, which clearly do not yield a reasonable $\lambda^{2}$ fit. A good fit is obtained if both the $2 \mathrm{~cm}$ and $1.3 \mathrm{~cm}$ polarization angles are rotated by $90^{\circ}$, with the implied offset between $\chi_{0}-\chi_{o p t} \simeq 60^{\circ}$. An inspection of the core degrees of polarization at our three wavelengths and the $2 \mathrm{~cm}-1.3 \mathrm{~cm}$ and $1.3 \mathrm{~cm}-7 \mathrm{~mm}$ core spectral indices $\left(\alpha \simeq 0.46, S \propto v^{\alpha}\right)$ provide no evidence for an optical-depth transition in our wavelength range, but suggest that the VLBA core is probably optically thick at all three wavelengths. Alternatively, a comparably good fit is obtained if the $1.3 \mathrm{~cm}$ polarization angle is rotated by $\pi$ and the $2 \mathrm{~cm}$ polarization angle by $2 \pi$; in this case, the inferred rotation measure is about $16,700 \mathrm{rad} / \mathrm{m}^{2}$ 

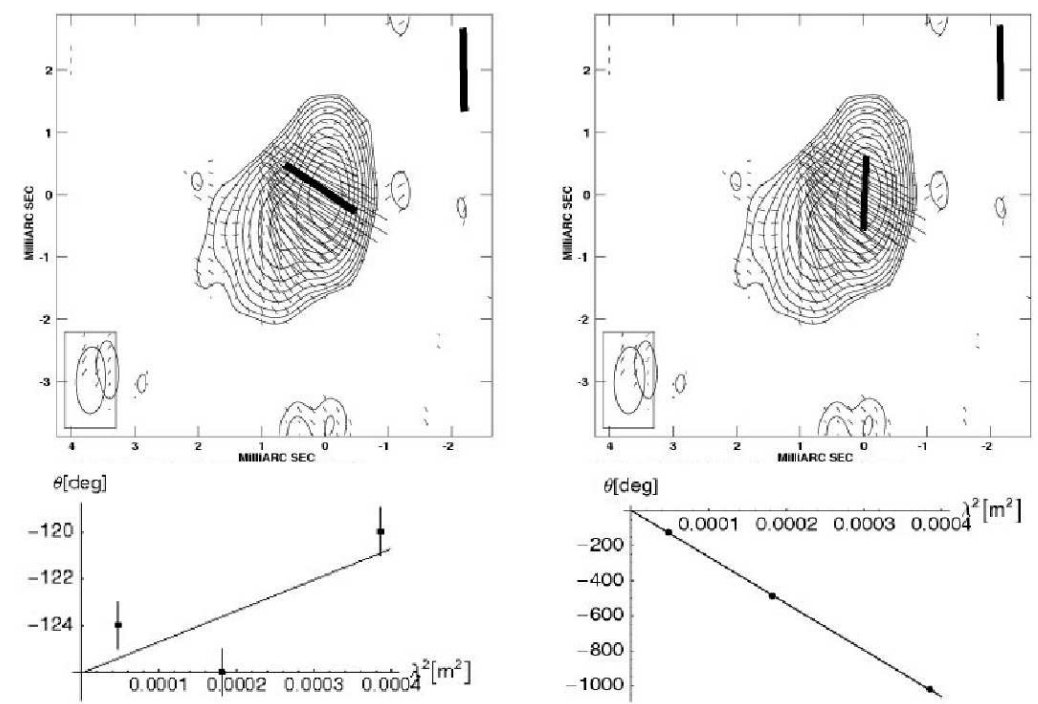

Figure 2: Two alternative rotation-measure fits for 2145+067. Left: the rotation-measure fit obtained for the nominal observed radio polarization angles. Right: another possible fit allowing for possible $n \pi$ ambiguities in the observed radio polarization angles. The thick lines in the corner and in the core show the optical and VLBI-core Faraday-corrected polarization angles, respectively.

and the inferred zero-wavelength polarization angle is $\chi_{0} \simeq 45^{\circ}$, offset from $\chi_{\text {opt }}$ by roughly $80^{\circ}$, close to the offset expected if the radio and optical emission regions are cospatial, but the radio core emission is optically thick.

Similarly, Fig. 2 shows two possible rotation-measure fits for $2145+067$. The left option shows the fit to the nominal observed radio-core polarization angles, which agree with each other to within about $6^{\circ}$. This fit is acceptable, and implies an offset $\Delta \chi \simeq 53^{\circ}$. However, if we add $2 \pi$ to the $1.3 \mathrm{~cm}$ and $5 \pi$ to the $2 \mathrm{~cm}$ polarization angles (right panels in Fig. 2), we obtain a comparably good fit that corresponds to a much larger but still reasonable core rotation measure of about $+47,000 \mathrm{rad} / \mathrm{m}^{2}$ and a zero-wavelength polarization angle of about $-74^{\circ}$. This fit implies a near alignment between the optical and VLBI-core polarization angles, $\Delta \chi \simeq 5^{\circ}$, which is consistent with an optically thin VLBI core ( $\alpha=-0.30$ between $2 \mathrm{~cm}$ and $1.3 \mathrm{~cm}$ and $\alpha=-0.60$ between $1.3 \mathrm{~cm}$ and $7 \mathrm{~mm}$ ).

We can find at least one acceptable fit with a high core rotation measure for essentially every source in our sample, usually implying rotation measures of the order of several tens of thousands of $\mathrm{rad} / \mathrm{m}^{2}$. In a few cases, we can identify an unambiguous best fit, but in others two or more different possible core rotation measures are plausible. Some of these fits imply intrinsic radio core polarization angles that are either aligned with or orthogonal to the optical polarization angle, consistent with the observed core spectral indices.

It can be argued that indiscriminately adding some number of $\pi$ rotations to the observed VLBA polarization angles, would eventually end in a good fit, even if the inferred rotation measure has no physical basis. For this reason, we held the $7 \mathrm{~mm}$ polarization angle fixed at its observed value, and considered rotations of no more than $\pm 5 \pi$ for the $2 \mathrm{~cm}$ polarization angles. The corresponding rotation measures range up to roughly $\pm 50,000 \mathrm{rad} / \mathrm{m}^{2}$, and are plausible, since we are probing regions of higher electron density and stronger magnetic fields. Similar rotation measures were found previously $[6,11]$. In addition, allowing for a high core rotation measure yields a good 
fit to the rotated polarization angles, there is no guarantee that the corresponding zero-wavelength core polarization angle will show some correlation with the optical polarization angle.

Therefore, it is likely that some of our alternative high core rotation meausures are correct, but they must be confirmed. It remains possible that internal Faraday rotation is influencing the observed polarization angles in some cores. Unfortunately, it is impossible to distinguish between the various scenarios with our current three-wavelength data. For this reason, we have obtained new VLBA polarization observations of 8 sources from our sample at 5 wavelengths from $7 \mathrm{~mm}$ to $2 \mathrm{~cm}$. The new data will improve our ability to identify high external Faraday rotation and the signature of internal Faraday rotation if present in the cores of these AGNs. Once we are able do uniquely determine the intrinsic polarization angle, we will be able to provide more quantitative statements about cospatiality and/or magnetic field structure between the optical and radio emitting parts of the base of the jet.

The possible presence of high core rotation measures has certain interesting implications. First, it would suggest that the physical conditions in the sub-parsec-scale jets in quasars are more extreme than has previously been thought. Second, a friendly warning for observers: we must be careful when observing polarization in quasar cores. The possible presence of high core rotation measures should be taken into consideration when planning polarization observations. For example, it is clear from our results that three wavelengths may not be sufficient to unambiguously derive reliable Faraday rotations, even at short VLBI wavelengths. In addition, it seems likely that we should not assume that the $7 \mathrm{~mm}$ polarization angles are a good aproximation to the intrinsic polarization, as has usually been assumed. Furthermore, many of the MOJAVE[12] $2 \mathrm{~cm}$ core polarization angles are likely subject to appreciable Faraday rotation.

\section{References}

[1] Algaba, J. C., Gabuzda, D. C., Smith, P.S., in High Energy Phenomena in Relativistic Outflows, International Journal of Modern Physics D, in press

[2] D'Arcangelo, F. D., Marscher, A. P., Jorstad, S. G., Smith, P. S., Larionov, V. M., Hagen-Thorn, V. A., Kopatskaya, E. N., Williams, G. G., Gear, W. K., 2007, ApJ, 659, L107

[3] Gabuzda, D. C., Rastorgueva, E. A., Smith, P. A., O'Sullivan, S. P., 2006, MNRAS 369, 1596

[4] Ghisellini, G., Maraschi, L., Treves, A., 1985, A\&A, 146, 204

[5] Jorstad, S. G., Marscher, A. P., Mattox, J. R., Allen, M. F., Aller, H. D., Wehrle, A. E., Bloom, S. D., 2001, ApJ, 556, 738

[6] Jorstad, S. G., Marscher, A. P., Stevens, J. A., Smith, P. S., Forster, J. R., Gear, W. K., Cawthorne, T. V., Lister, M. L., Stirling, A. M., Gómez, J. L., Greaves, J. S., Robson, E. I, 2007, AJ 134, 799

[7] Marscher, A. P., Gear, W. K., 1985, ApJ 298114

[8] Moore, R. L. \& Stockman, H. S., 1984, ApJ, 279, 465

[9] Schmidt, D. G., Stockman, H. S., Smith, P. S., 1992, ApJ 398, L57

[10] Smith, P. S., Williams, G.G., Schmidt, G.D., Diamond-Stanic, A.M. \& Means, D. L., 2007, ApJ, 663, 118

[11] Zavala, R. T. \& Taylor, G. B., 2004 ApJ, 612, 749

[12] http://www.physics.purdue.edu/MOJAVE/ 